this, new ultrasound machines were purchased and ultrasonographers received training to perform outlet views of the fetal heart as well as 4 chamber views.

Aims The aims of this audit were 1) to establish whether this intervention has led to improved antenatal detection of significant $\mathrm{CHD}$ and 2) to compare these detection rates with national figures.

Methods Antenatal detection rates of CHD were again audited for all babies who received an anomaly scan in Jan 2009Dec 2010. Children with significant CHD were identified through their attendance at cardiac clinic. We then looked retrospectively at each child's anomaly scan $(18+0-20+6$ weeks gestation) and recorded whether or not a cardiac lesion had been detected antenatally. We also reviewed data from the South West Congenital Anomoly Register (SWCAR) and local electronic and paper records by which method we were able to include antenatally detected significant CHD in foetuses who did not survive.

Results Following the intervention antenatal detection of significant $\mathrm{CHD}$ rose to $72 \%$. This compares with national detection rates of around $30 \%$ over the same period.

Conclusion Our findings confirm the necessity of cardiac outlet views along side 4-chamber views as part of antenatal anomaly scanning with good supervision and support from local and regional Fetal Medicine Specialists.

\section{G171(P) PROVIDING PSYCHOLOGY SUPPORT IN A DISTRICT PAEDIATRIC CARDIOLOGY SERVICE}

${ }^{1} \mathrm{C}$ Illingworth, ${ }^{2} Y$ Singh, ${ }^{2} \mathrm{~W}$ Kelsall. ${ }^{1}$ Department of Clinical Psychology, Cambridge University Hospitals NHS Foundation Trust, Cambridge, UK; '² Department of Neonatology and Paediatric Cardiology, Cambridge University Hospitals NHS Foundation Trust, Cambridge, UK

\subsection{6/archdischild-2015-308599.166}

Introduction Advances in medical and surgical care have improved mortality and morbidity rates in children with congenital heart disease (CHD). It has been recognised that children with CHD and their families benefit from psychology input. In 2010 the National Reference Group for Psychologists working in Paediatric Cardiology drafted minimum standards for services to meet the psychosocial needs of these children. These were approved by the British Psychological Society, the Congenital Heart Service Standards Group and the Clinical Implementation Advisory group in 2013.

Aims The study reviewed the impact of newly introduced clinical psychology service for Paediatric Cardiology patients in Cambridge.

Methods Patients were identified from local paediatric and psychology databases. Children and families were referred to a Clinical Psychologist working in general paediatrics. Access to the service was initially limited the children referred were deemed to be the most complex in the Service.

Results Between March 2012 and March 2014 twenty five referrals were made and 18 patients/families were seen, 2 declined input and 5 are pending. Of those seen, 10 were male and 8 female aged between 4 months and 16 years. There were 116 contacts with the psychologist. Most were offered 6 appointments, ranging from 2 to 20. The reasons for referral were for: parental anxiety/trauma (4), attachment issues (1), adjustment to a new diagnosis (4), management of neurodevelopmental concerns and school difficulties (1), behavioural difficulties (2) and emotional difficulties (6). So far, eleven Service satisfaction questionnaires have been sent to 11 families and six have been returned. All respondents valued accessing a psychologist locally rather than travelling to a specialist centre.

Conclusions This study supports national and local drivers to provide high quality medical and psychological care close to child's home from birth through to transition to adult services. There is anecdotal evidence that direct family contact with the psychologist has freed up Consultant time and improved shared care. This data together with the standards documents have been used to establish a dedicated cardiology psychology service.

\section{G172(P) CASE REPORT:CYTOMEGALOVIRUS AND KAWASAKI DISEASE - IS THERE A LINK?}

KL Kok, TD Jones. Paediatrics, Peterborough City Hospital, Peterborough, UK

\subsection{6/archdischild-2015-308599.167}

Aims Kawasaki disease is an uncommon cause of fever in a young infant (less than 6 months) and is associated with lifethreatening complications. Cytomegalovirus has been linked with atherosclerosis of coronary arteries in some adult studies. Acute Cytomegalovirus infection has been detected in a few reported cases of Kawasaki disease in infants.

Methods A retrospective case review was done of a 3 month old Asian infant, who presented to a district general hospital, with a 3 day history of fever and irritability. She was treated with intravenous antibiotics for presumed sepsis. The fever persisted after 5 days and she was transferred to a tertiary hospital for further investigations into the cause of fever.

Results Cytomegalovirus DNA was detected by polymerase chain reaction (PCR) in the infant's blood. Cytomegalovirus IgM was not detected. ECG and echocardiogram were not performed due to lack of clinical evidence of underlying cardiac cause at the time. The fever was settling and infection markers were improving. The infant looked to be improving, according to the parents. The infant became acutely unwell, arrested and passed away on day 13 of illness.

At post-mortem, multiple thrombosed giant aneurysms (up to $17 \mathrm{~mm}$ ) of the coronary arteries were found. There were multiple saccular coronary artery aneurysms affecting the right coronary $(11 \times 17 \mathrm{~mm})$, left descending coronary artery, left diagonal artery, left circumflex and the right posterior descending artery. The pericardial cavity contained $70 \mathrm{mls}$ of blood. There was moderate to severe coronary artery arteritis. The cause of death was cardiac tamponade secondary to rupture of a coronary artery aneurysm, caused by Kawasaki disease. Cytomegalovirus DNA was detected in liver and lung tissue.

Conclusion Kawasaki disease is an uncommon but important differential in a young infant with fever and requires prompt treatment with immunoglobulins and Aspirin. If echocardiography is not available, an ECG may provide useful information such as ST segment changes and Q waves. The presence of Cytomegalovirus DNA on blood PCR raises the possibility of Kawasaki disease and a positive result may be helpful additional information in the diagnosis of Kawasaki disease. 